\title{
Evaluación de tres métodos para la recuperación de metales pesados en fertilizantes
}

\author{
Esteban Pérez López \& César Barrantes Murillo \\ Universidad de Costa Rica, Recinto de Grecia, Costa Rica; esteban.perezlopez@ucr.ac.cr; cesbar27@hotmail.com
}

Recibido 16-II-2017 • Corregido 26-VI-2017 • Aceptado 07-VII-2017

\begin{abstract}
Evaluation of three methods for the recovery of heavy metals in fertilizers. Fertilizers are essential for agriculture but can produce contamination with heavy metals and affect the health of humans and farm animals. Reliable methods to measure these metals are important.We evaluated the recovery of the analytical method employed in a services laboratory in Costa Rica for the quantification of nickel, chromium, cadmium, lead, arsenic, selenium and mercury in fertilizers. A phosphate and a nitrogenous matrix were studied separately, enriched at three concentration levels within the working range, to establish the accuracy of the analysis procedure based on AOAC for the validation of analytical methods (80-110\%). In the quantitative study both cadmium and chromium for the two matrices presented optimum results from the point of view of recovery; lead also had positive accuracy (except at low concentration). On the other hand, nickel in the phosphate matrix was slightly above the maximum permissible limit, but not the nitrogen, Finally in the metals arsenic, selenium and mercury there was no recovery for both samples using the established procedure.
\end{abstract}

Keywords: Heavy metals, atomic absorption, acid digestion, recovery.

En los países en desarrollo, la mayoría de los agricultores son de pequeña escala y forman parte de la pobreza rural. Dado que la mejora en la productividad resulta en más alimentos e ingresos, se han desarrollado los fertilizantes (Roma, 2002). Este se define como cualquier material orgánico u inorgánico, natural o sintético que proporciona a las plantas, uno o más elementos químicos para su normal crecimiento (Morel, 2003). Actualmente son ampliamente utilizados ya que permiten producir más alimentos y cultivos comerciales, y de mejor calidad. Con los fertilizantes se puede mejorar la baja fertilidad de los suelos que han sido sobreexplotados, y a lo largo de la historia son pieza clave para optimizar las técnicas
RESUMEN: Los abonos son fundamentales para la agricultura pero pueden producir contaminación con metales pesados que afectan la salud humana y de animales domésticos, por lo que es necesario tener métodos fiables para medir esos metales. Evaluamos la recuperación del método analítico empleado en un laboratorio de servicios en Costa Rica para la cuantificación de níquel, cromo, cadmio, plomo, arsénico, selenio y mercurio en fertilizantes. Estudiamos por separado una matriz fosfatada y otra nitrogenada, las cuales fueron enriquecidas a tres niveles de concentración determinadas dentro del rango de trabajo, para establecer la exactitud del procedimiento de análisis basándose en lo establecido por la AOAC para validación de métodos analíticos (80-110\%). En el estudio cuantitativo tanto el cadmio como el cromo para las dos matrices presentaron resultados óptimos desde el punto de vista de recuperación; el plomo de igual forma tuvo una exactitud positiva excepto a niveles bajos de concentración; por otra parte el níquel en la matriz fosfatada se encontró ligeramente por encima del límite máximo admisible, no así la nitrogenada; y por último en los metales arsénico, selenio y mercurio no hubo recuperación para ambas muestras usando el procedimiento establecido.

Palabras clave: Metales pesados, absorción atómica, digestión ácida, recuperación. de cultivo y promover el bienestar de las personas y países que dependen de este tipo de prácticas económicas (Finck, 2000).

Los fertilizantes contienen nutrientes para las plantas y pueden fácilmente incorporarse al suelo para escalar su fertilidad neutral, su modo de acción al cubrir las necesidades de los vegetales es directo, como las enmiendas que modifican las propiedades del suelo como estructura o pH (Navarro, 2014). En este tipo de productos son vitales los elementos esenciales de las plantas, los cuales se pueden dividir en nutrientes principales $(N, P, K)$, secundarios ( $\mathrm{S}, \mathrm{Na}, \mathrm{Ca}, \mathrm{Mg})$ y micronutrientes $(\mathrm{Co}, \mathrm{Fe}, \mathrm{Cu}, \mathrm{B}$, $\mathrm{Mn}, \mathrm{Zn}$ ) (Thompson \& Trohe, 2007). 
La actividad agrícola de Costa Rica ha sido una fuente de ingresos muy importante, principalmente por las plantaciones de banano y café, pero por otro lado las malas prácticas en el uso de agroquímicos han provocado contaminaciones serias, por ejemplo, durante más de 20 años, a partir del año 1930, las plantaciones bananeras fueron sometidas a tratamientos con el fungicida "Caldo Bordolés" el cual posee un alto contenido de cobre; lamentablemente estas actividades ocasionaron el deterioro de terrenos de un alto potencial agrícola. (Alvarado \& Romero, 2010).

Existen estudios que demuestran la importancia del control de los metales pesados para el medio ambiente, uno de ellos es el llamado: Aspectos bioquímicos y genéticos de la tolerancia y acumulación de metales pesados en plantas, en donde se desgrana cuáles son los elementos clave que la genética molecular de plantas hace participar en la tolerancia y acumulación de metales pesados (Aguilar \& Navarro, 2007).

En el artículo de González, Prieto, Román \& Solís (2009), se alerta acerca de la contaminación y fitotoxicidad de los metales pesados provenientes del suelo y el agua, estableciendo que pueden llegar a ser perjudiciales para la salud animal y humana.

En lo referente a la evaluación de la recuperación, existen diferentes artículos destinados a establecer metodologías para medir la concentración de metales pesados. Entre ellos está la validación de la metodología para el análisis de metales pesados en muestras de aguas y aguas residuales por la técnica de absorción atómica; en la que se asevera la necesidad de evaluar la recuperación del método para poder validar un método (Gómez, 2011).

En estudios relacionados, Robledo (2012) de la Universidad Tecnológica de Pereira, destaca el estudio en análisis de aguas donde se validaron metodologías para la cuantificación de cadmio por la técnica de horno de grafito. En el sector agrícola también se han llevado a cabo estudios como el de Saucedo, Sierra, Zaldívar \& Zúñiga (2005), para los metales sodio, potasio, cadmio, mercurio y plomo en plantaciones de caña, producto muy común en Costa Rica.

En otros estudios, se aplican metodologías para evaluar la recuperación que evidencian que los procedimientos para verificar dicho parámetro son muy comunes, y respaldan la validez de lo ensayado. El estudio de Ávila, Lira \& Padilla (1997) investigó una metodología para la determinación de trazas de los herbicidas 2,4-D y 2,4-DB, en el que se llevaron a cabo pruebas de recuperación mediante la inyección de cantidades de soluto conocidas, fortificando los herbicidas, aspecto clave en la optimización.
Muy similar al anterior, el estudio de extracción enzimática de arsénico en muestras de orígen vegetal, mediante pruebas de recuperación adicionando enzimas y variando los medios de extracción, se establece un método alterno para el procedimiento. Las metodologías para evaluar la recuperación mejoran y generan alternativas, pero en síntesis se maneja el mismo principio de trabajo (Ayala, Garro \& Gimenez, 1999).

Por su parte los autores Acosta, Flores, Senior \& Toyo (2006), estudió la evaluación de metales pesados en tres residuos orgánicos de diferente naturaleza en donde, ni en el lodo residual, estiércol o residuos industriales, se hallaron cantidades potencialmente peligrosas.

Finalmente, Guerrero (2010), elaboró un estudio del efecto matriz en el análisis de plaguicidas y agroquímicos por la técnica de cromatografía de gases en la cual se determinó que existe una variación en las señales cromatográficas y los resultados, por interferencias derivadas de la matriz. También, en Ecuador se valida un método para análisis de metales pesados en diferentes matrices de suelos, que evidenció el efecto diverso que pueden tener las matrices en un método analítico, en este caso con suelos de diferentes fuentes agrícolas (Remache, 2013).

En Costa Rica, el laboratorio de análisis ambiental de la Universidad Nacional elaboró la validación de un método de análisis para la determinación de metales pesados en partículas $\mathrm{PM}_{10}$ colectadas en aire ambiente, a partir de muestras recolectadas en la ciudad de San José y por medio de la técnica espectrofotometría de absorción atómica con horno de grafito. Dicha investigación utilizó un método de recuperación muy similar al empleado en este estudio, y al basarse en normativa costarricense reviste mayor importancia (Herrera \& Román, 2010).

La calidad de un fertilizante está definida por algunas propiedades físicas (granulometría, aptitud para mezclas físicas) y químicas que tengan relevancia agronómica; esta última sección se ve relacionada con análisis de acidez, alcalinidad, pH y composición de los nutrientes y otras sustancias o compuestos químicos presentes en el producto (Laínez, 2005).

Los metales pesados, debido a su toxicidad y su tendencia a acumularse en los sistemas biológicos, representan un riesgo para la salud humana y los ecosistemas, aun en bajas concentraciones. Dichos elementos provienen de diferentes fuentes: combustibles, aerosoles urbano-industriales, desechos líquidos y sólidos de origen animal y humano, industria minera, química y textil, etc. En el sector agropecuario son fuentes contaminantes los cohetes antigranizo, aguas residuales utilizadas para riego, guanos, compost, plaguicidas y fertilizantes (Burba, Cavagnaro \& Martí, 2010). 
Los suelos agrícolas por efecto de la actividad humana $\mathrm{u}$ otras causas pueden llegar a acumular progresivamente uno o varios de estos elementos hasta alcanzar niveles altamente tóxicos, cuando son absorbidos por la planta y posteriormente por el hombre o animal (Lázaro, 2008).

El método analítico más utilizado para la cuantificación de metales pesados es la espectrometría de absorción atómica a raíz de su rapidez, fiabilidad, sencillez y economía; ya sea por los métodos de llama, horno de grafito o generador de hidruros (Zúñiga, 2005; Reyes \& Walton, 2001). Por absorción atómica se determina cadmio, cromo, cobre, plomo, níquel y zinc, por llama; otros como arsénico y selenio debido a las bajas cantidades se analizan por medio de horno de grafito; y para elementos muy volátiles como el mercurio se opta por el acople de generador de hidruros (Ramos, 2009). En relación a los parámetros de validación o verificación estos deberán determinarse de acuerdo al tipo de método y entre ellos se encuentran la selectividad, linealidad, sensibilidad, limites, exactitud, precisión, robustez y aplicabilidad (Gómez, Pérez \& Sierra, 2009). La recuperación esta intrínsecamente relacionada a las características de la matriz de la muestra (Holler, Skoog \& West, 2010).

El presente estudio, tuvo como fin primordial la evaluación de la recuperación de metales pesados para métodos analíticos en fertilizantes por espectroscopía de absorción atómica en llama, horno de grafito y generador de hidruros.

\section{METODOLOGÍA}

El procedimiento analítico para metales pesados en muestras de fertilizantes, incluyó la digestión de la muestra para reducir interferencias y aislar el elemento de interés ( $2 \mathrm{~g}$ en balones aforados de $100 \mathrm{~mL}$ ) con $5 \mathrm{~mL}$ de ácido clorhídrico, por medio de columnas de condensación a una temperatura de $200^{\circ} \mathrm{C}$ por 30 minutos; una vez digerida se aforó y midió según la técnica correspondiente (AA), en comparación con la curva de calibración elaborada para cada metal implicado, en cinco niveles de concentración, en los rangos mostrados en el Cuadro 1. El método para análisis de metales pesados fue basado en lo que establece la Agencia de Protección del Medio Ambiente de Estados Unidos de Norteamérica (EPA); sin conocer la recuperación para la metodología en matrices fosfóricas y nitrogenadas (Agroanálisis Costa Rica, 2016).

Para evaluar la recuperación, se utilizó el método adición/recuperación, el cual consiste en enriquecer una muestra adicionando cantidades conocidas del analito. En este caso se realizó para el níquel, cromo, cadmio, plomo, arsénico, selenio y mercurio en dos matrices de fertilizantes diferentes (fosforada y nitrogenada), una vez determinado el contenido promedio se enriquecen muestras a tres niveles de concentración por triplicado. En el Cuadro 2 se detalla el contenido teórico para los metales pesados en ambas matrices.

Conocidos los valores teóricos se procedió a enriquecer las matrices según los factores antes establecidos. En el Cuadro 3 se despliegan las concentraciones para cada metal, a las cuales fueron enriquecidas tanto la matriz fosfatada como nitrogenada.

Debe contemplarse que los niveles de concentración se plantean dentro del rango de la curva de calibración del método (Cuadro 1).

Para el análisis de los metales se utilizó la técnica de espectroscopia de absorción atómica con llama para níquel, cromo, cadmio, plomo; horno de grafito para arsénico, selenio; y generador de hidruros para mercurio. El equipo utilizado es marca Perkin Elmer, provisto con automuestreador y utilizando tanto la lámpara de cátodo

\section{CUADRO 2}

Concentraciones teóricas de matrices estudiadas ( $\mathrm{mg} / \mathrm{L}$ y $\mu \mathrm{g} / \mathrm{L}$ )

\begin{tabular}{|c|c|c|c|c|c|c|c|}
\hline Matriz & $\begin{array}{c}\mathrm{Ni} \\
\pm 0,001 \mathrm{mg} / \mathrm{L}\end{array}$ & $\begin{array}{c}\mathrm{Cr} \\
\pm 0,001 \mathrm{mg} / \mathrm{L}\end{array}$ & $\begin{array}{c}\mathrm{Pb} \\
\pm 0,001 \mathrm{mg} / \mathrm{L}\end{array}$ & $\begin{array}{c}\mathrm{Cd} \\
\pm 0,001 \mathrm{mg} / \mathrm{L}\end{array}$ & $\begin{array}{c}\text { As } \\
\pm 0,5 \mu \mathrm{g} / \mathrm{L}\end{array}$ & $\begin{array}{c}\mathrm{Se} \\
\pm 0,01 \mu \mathrm{g} / \mathrm{L}\end{array}$ & $\begin{array}{c}\mathrm{Hg} \\
\pm 0,01 \mu \mathrm{g} / \mathrm{L}\end{array}$ \\
\hline Matriz Fosforada & 0,290 & 2,300 & 0,102 & 0,089 & 249,2 & 2,20 & 2,60 \\
\hline Matriz Nitrogenada & 0,323 & 1,770 & 0,042 & 0,080 & 239,1 & 1,90 & 1,01 \\
\hline
\end{tabular}


CUADRO 3

Concentraciones enriquecidas para evaluación de recuperación.

\begin{tabular}{|c|c|c|c|}
\hline Metal & Nivel 1 & Nivel 2 & Nivel 3 \\
\hline \multicolumn{4}{|c|}{$( \pm 0,01$ ppm) } \\
\hline Níquel & 0,50 & 1,00 & 1,50 \\
\hline Cadmio & 0,50 & 1,00 & 1,50 \\
\hline Cromo & 1,00 & 3,00 & 5,00 \\
\hline Plomo & 2,00 & 5,00 & 8,00 \\
\hline \multicolumn{4}{|c|}{$( \pm 0,1 \mathrm{ppb})$} \\
\hline Arsénico & 30,0 & 50,0 & 80,0 \\
\hline Selenio & 30,0 & 50,0 & 80,0 \\
\hline Mercurio & 10,0 & 30,0 & 40,0 \\
\hline
\end{tabular}

hueco, como la de descarga de electrones, por la misma variedad en la naturaleza de las especies en estudio. Una vez insertadas las muestras, las especies metálicas son identificadas y cuantificadas por interpolación con las curvas de calibración. Los reactivos empleados: $\mathrm{HCl}$ (37\%), agua destilada y los respectivos estándares certificados para cada metal.

Por último, para cada uno de los casos de estudio, se calculó el porcentaje de recuperación y la desviación estándar relativa (DSR) para evidenciar o no, la precisión entre los valores experimentales medidos en cada caso.

\section{RESULTADOS}

Al evaluar la recuperación de los metales pesados níquel, cromo, cadmio, plomo, arsénico, selenio y mercurio, para el método analítico de fertilizantes nitrogenados y fosfatados por espectroscopía de absorción atómica en llama, horno de grafito o generador de hidruros. Los resultados se resumen en los Cuadros 4 y 5 en los cuales se despliegan tanto los valores de recuperación como los de DSR (desviación estándar relativa) para los metales analizados en las matrices fosfatadas y nitrogenadas. Es importante destacar que para estos dos parámetros se siguen los criterios de aceptación establecidos por la AOAC para la validación de métodos analíticos donde \%R: 80-110\% y DSR: > 2\% (Sandoval, 2010).

Específicamente, en el Cuadro 4 se detallan los resultados obtenidos para la recuperación ensayada en la matriz fosfatada, así como los respectivos desvíos estándar relativos (DSR\%).

De igual forma, el cuadro 5 presenta los resultados obtenidos para la recuperación ensayada en la matriz

CUADRO 4

Resultados de recuperación y precisión para la matriz fosfatada.

\begin{tabular}{|c|c|c|c|}
\hline Metal & Nivel de concentración enriquecido & \% de Recuperación & DSR (\%) \\
\hline \multirow[t]{3}{*}{ Níquel } & Nivel 1 & 120 & 3,15 \\
\hline & Nivel 2 & 118 & 3,15 \\
\hline & Nivel 3 & 119 & 2,42 \\
\hline \multirow[t]{3}{*}{ Cadmio } & Nivel 1 & 105 & 0,55 \\
\hline & Nivel 2 & 101 & 0,57 \\
\hline & Nivel 3 & 98 & 0,59 \\
\hline \multirow[t]{3}{*}{ Cromo } & Nivel 1 & 91 & 1,90 \\
\hline & Nivel 2 & 95 & 1,50 \\
\hline & Nivel 3 & 97 & 1,03 \\
\hline \multirow[t]{3}{*}{ Plomo } & Nivel 1 & 115 & 1,81 \\
\hline & Nivel 2 & 107 & 0,54 \\
\hline & Nivel 3 & 105 & 0 \\
\hline \multirow[t]{3}{*}{ Arsénico } & Nivel 1 & 0 & 0 \\
\hline & Nivel 2 & 0 & 0 \\
\hline & Nivel 3 & 0 & 0 \\
\hline \multirow[t]{3}{*}{ Selenio } & Nivel 1 & 0 & 0 \\
\hline & Nivel 2 & 0 & 0 \\
\hline & Nivel 3 & 0 & 0 \\
\hline \multirow[t]{3}{*}{ Mercurio } & Nivel 1 & 0 & 0 \\
\hline & Nivel 2 & 5 & 23 \\
\hline & Nivel 3 & 9 & 6,66 \\
\hline
\end{tabular}


CUADRO 5

Resultados de recuperación y precisión para la matriz nitrogenada.

\begin{tabular}{lccc} 
Metal & Nivel de concentración enriquecido & \% de Recuperación & DSR (\%) \\
Níquel & Nivel 1 & 108 & 3,74 \\
& Nivel 2 & 102 & 1,49 \\
Cadmio & Nivel 3 & 98 & 1,17 \\
& Nivel 1 & 99 & 0 \\
Nivel 2 & 98 & 0,59 \\
Cromo & Nivel 3 & 96 & 0 \\
& Nivel 1 & 85 & 2,71 \\
\multirow{4}{*}{ Plomo } & Nivel 2 & 85 & 1,18 \\
& Nivel 3 & 83 & 0,70 \\
& Nivel 1 & 125 & 0,79 \\
Arsénico & Nivel 2 & 109 & 0,53 \\
& Nivel 3 & 103 & 0 \\
& Nivel 1 & 12 & 5,03 \\
Selenio & Nivel 2 & 15 & 3,71 \\
& Nivel 3 & 22 & 21,01 \\
& Nivel 1 & 24 & 0,70 \\
Mercurio & Nivel 2 & 19 & 14,8 \\
& Nivel 3 & 28 & 0 \\
& Nivel 1 & 0 & 5,03 \\
\hline
\end{tabular}

nitrogenada, así como los respectivos desvíos estándar relativos (DSR\%).

\section{DISCUSIÓN}

Elementos analizados en llama: como se observa en los Cuadros 4 y 5 , tanto en la matriz fosforada como nitrogenada, para el cadmio se obtuvieron valores aceptables, debido a que la recuperación se mantuvo muy cercana al $100 \%( \pm 5 \%)$, y la precisión fue menor al $2 \%$ entre las réplicas en cada nivel de concentración. Por otra parte, los resultados para el cromo fueron muy buenos, satisfaciendo los criterios de aceptación establecidos, lo cual evidencia que el método analítico es exacto y preciso, prácticamente para cualquier punto de la curva de calibración de ambos metales. Ambos casos se evidencian en la figura 1.

En cuanto al níquel, la matriz fosfatada presentó resultados fuera de especificación como se muestra en la figura 2, de hecho en ningún nivel de concentración recuperó de manera óptima, con la tendencia de siempre estar por arriba del $110 \%$ establecido como máximo admisible. Es destacable que aunque tampoco sobrepasó el rango por mucho, los datos recolectados siempre fueron mayores a lo que se tenía estimado teóricamente; de igual manera el DSR sobrepasó los valores esperados para un buen análisis. Lo anterior demuestra que la capacidad que tiene el método analítico para determinar cuantitativamente la especie química que ha sido adicionada a la muestra no es la ideal, en el caso de la matriz fosforada.

Como se observa, para la matriz nitrogenada los resultados si evidenciaron una recuperación óptima para el níquel, por lo que, basado en los resultados el método no es funcional para matrices fosfatadas de fertilizantes. Esto puede ser debido al efecto de la matriz, el cual consiste en una disminución o aumento de la respuesta instrumental del analito debido a la presencia de otros componentes. En otras palabras, para la misma concentración de analito, el análisis de una muestra real o de una disolución estándar del analito puro no proporciona la misma respuesta instrumental (Harris, 2006).

Las interrupciones por la matriz son debidas a la influencia que tiene el entorno que rodea a los átomos en estado fundamental que se pretende analizar y pueden llegar a generar interferencias por traslape de bandas moleculares, las cuales se materializan en el realce o disminución de las señales emitidas por el instrumento. 

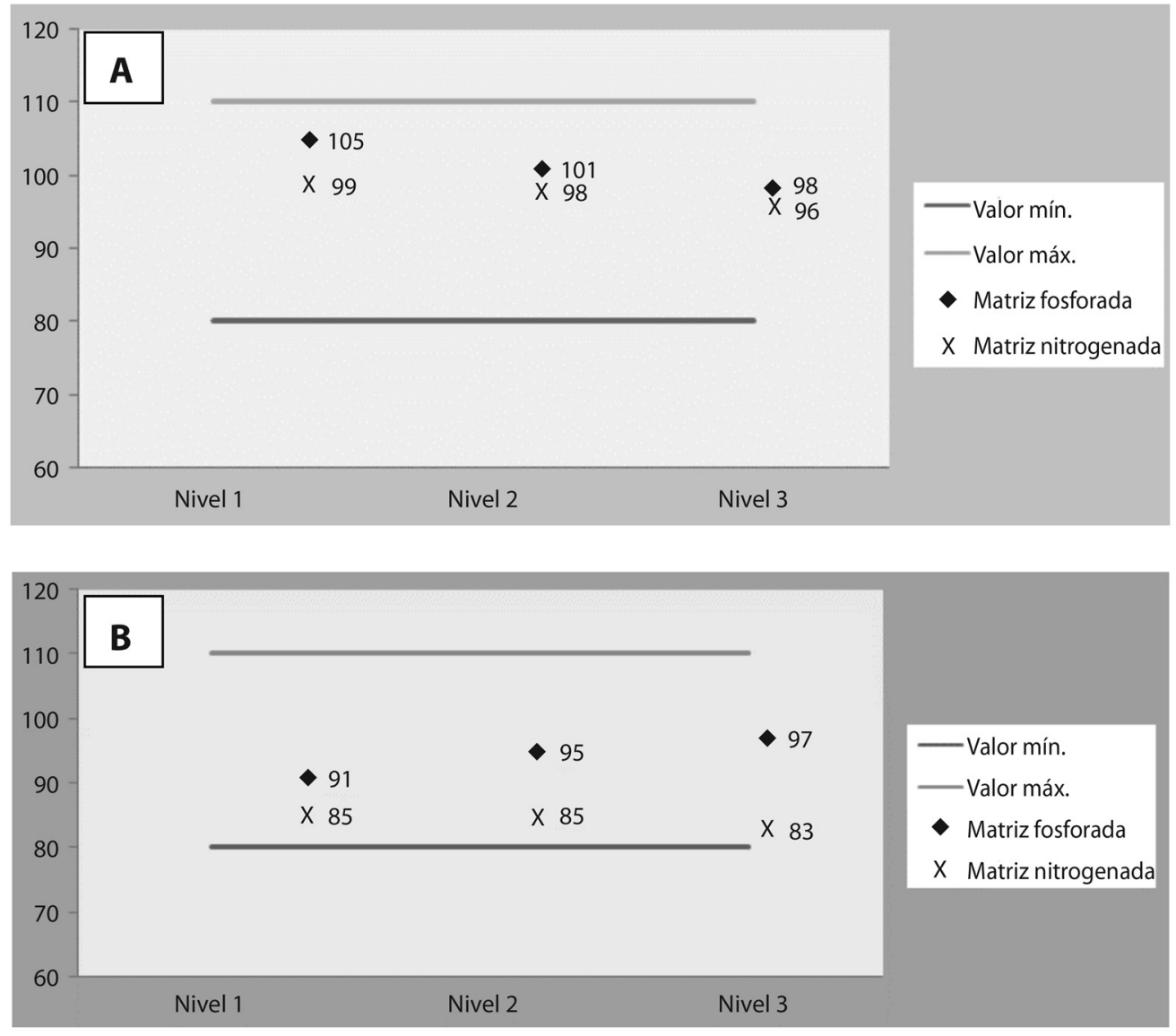

Fig. 1. Porcentaje de recuperación de cadmio (A) y cromo (B).

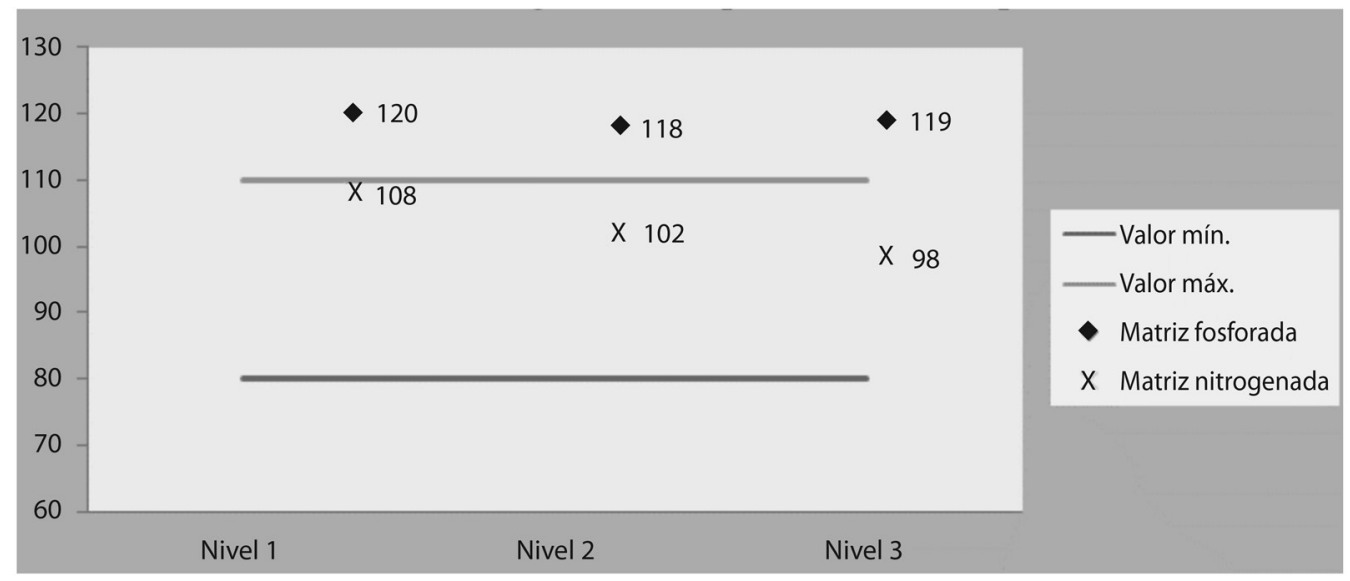

Fig. 2. Porcentaje de recuperación de níquel. 
Para evaluar si realmente los resultados son generados por un tema del contenido de la matriz se puede llevar a cabo un blanco de muestra (tal como se hizo al contemplar la concentración cero en los rangos) con el cual se verifique si existe realmente una relación para obtener datos negativos desde el punto de vista de recuperación (Casanova, 2007).

De igual forma no se pueden obviar problemas instrumentales, que con frecuencia suelen aparecer. Los métodos de absorción son muy específicos debido a que las líneas de absorción son notablemente estrechas $(0,002$ a $0,005 \mathrm{~nm}$ ) y porque las energías de transición electrónicas son únicas para cada elemento, de ahí se genera la necesidad de contar con lámparas específicas para las especies a cuantificar (Manzano, 2003). La eficiencia de este componente va depender de su voltaje, cuando estos comienzan a fallar, los resultados evidencian una pobre linealidad $(<0,995)$, baja precisión y exactitud e inconsistencia en los resultados (interferencias de emisión); lo que ocurrió con el níquel en la matriz fosforada, a diferencia de los demás metales.

Por último en cuanto a los metales de llama, se encuentra el plomo en donde se obtuvo un comportamiento constante para ambas matrices, como se puede apreciar en los Cuadros 4 y 5, la precisión para los 3 niveles de concentración es muy buena, manteniendo el DSR por debajo del $2 \%$, sin embargo, para la recuperación ambas matrices tienen problemas a niveles bajos de concentración (Fig. 3), presentando porcentajes de recuperación por encima del $110 \%$ aceptable; no así para los otros dos niveles de contenido de plomo, donde se mantienen dentro del rango 80-110\%.

Esta situación se puede generar a raíz de que las concentraciones enriquecidas son muy bajas $(2,00 \mathrm{ppm})$ y se acercan mucho al límite de cuantificación. El límite de cuantificación, se define como la cantidad más pequeña de un analito que se pueda cuantificar confiablemente por el instrumento, en este caso aún en niveles bajos de la curva de calibración, la medición presenta un sesgo importante (Arderiu, 1997). En este caso, las concentraciones son aceptables según la Norma de Fertilizantes y Enmiendas para uso Agrícola de Costa Rica (RTCR 485:2016) en donde se establece como límite máximo admisible de plomo $250 \mathrm{mg} / \mathrm{kg}$ (5ppm).

Con respecto a las desviaciones estándar relativas obtenidas es que normalmente dicho parámetro de precisión desciende conforme se aumenta el nivel de concentración enriquecido, cabe destacar que no se obtuvieron valores alarmantes en ningún nivel. Como antes se indicó al estar más cercano al límite de cuantificación del método se da un aumento de las interferencias y por ende variaciones entre resultados. Para corroborar lo anterior se elaboraron muestras para ambas matrices enriquecidas con niveles inferiores de la curva de calibración tanto para níquel como cromo; en esos casos las DSR tuvieron valores de 6-13\% para níquel y hasta un $43 \%$ para cromo, lo que comprueba que a bajos niveles de metales pesados (por debajo de la curva de calibración) no existe precisión alguna.

En temas de linealidad, el criterio de aceptación cualitativo que se usa para determinar la linealidad es el coeficiente de correlación (Metas y metrólogos asociados, 2008). En este caso se esperaba que el coeficiente de correlación fuera mayor o igual a 0,995; dicho criterio se logró cumplir para todos los análisis realizados en el estudio de linealidad, sin embargo, debe destacarse que elementos como el níquel presentaron problemas ocasionales en este sentido.

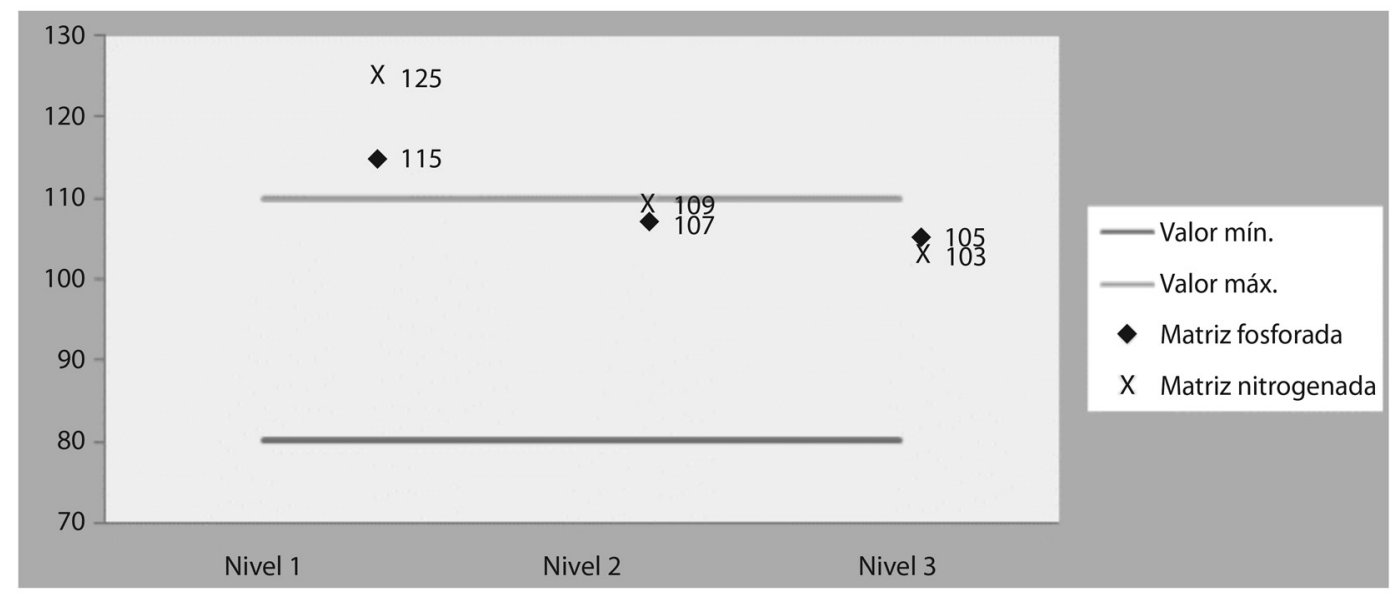

Fig. 3. Porcentaje de recuperación de plomo. 
Elementos analizados en horno de grafito y generador de hidruros: como se observa en los Cuadros 4 y 5 en términos de recuperación los valores son muy bajos o inclusive nulas del todo.

En este caso no es posible considerar una falla instrumental (a diferencia del níquel con la matriz fosforada), debido a tres razones principales: 1 ) la linealidad siempre fue ideal, inclusive mejor que en muchos de los análisis por la técnica de llama, lo que implícitamente demuestra que los patrones eran cuantificados con exactitud, 2) el equipo arrojaba concentraciones características adecuadas, y este es un parámetro importante utilizado para verificar la respuesta del instrumento, 3 ) las muestras utilizadas como control eran medidas a la perfección con una exactitud del $\pm 10 \%$.

A raíz de los descartes de problemas con el equipo, se llegó a la conclusión que la problemática radicó en el método en sí, simplemente no es adecuado para la cuantificación de selenio, arsénico y mercurio en las matrices consideradas en el estudio. Específicamente el problema se encuentra en la digestión, por el uso del ácido clorhídrico. Una preocupación importante cuando se digieren muestras, es la posibilidad de que una porción del analito pueda volatilizarse y perderse. Un gran número de compuestos forman cloruros volátiles que se pierden parcial o completamente de las disoluciones de ácido clorhídrico calientes. Entre estos elementos están los cloruros de estaño (IV), germanio (IV), antimonio (III), arsénico (III), hierro (III) y mercurio (II). Los oxicloruros de selenio y telurio también se volatilizan en cierto grado del ácido clorhídrico caliente (Skoog, Holler \& West, 2015).

Lo anterior explicaría el por qué no se está recuperando prácticamente nada de esos metales, también señala que el ácido puede formar sales insolubles con la plata y mercurio, inclusive se destaca que el $\mathrm{HCl}$ es un excelente disolvente para muestras inorgánicas, sin embargo, su aplicación en muestras orgánicas es limitada por lo cual simplemente no es viable utilizarlo en análisis de fertilizantes o agroquímicos.

Dada la severidad que implica tener un método que no cuantifique metales pesados tan nocivos y peligrosos para el medio ambiente y salud de cultivos, animales e inclusive humanos, se debe sustituir el ácido clorhídrico por ácido nítrico y llevar a cabo pruebas para verificar la efectividad del cambio. El $\mathrm{HNO}_{3}$ es un reactivo con alto poder oxidante y es ampliamente recomendado para matrices o muestras orgánicas y las únicas desviaciones que teóricamente puede presentar se dan con el oro, platino y aluminio debido a que parte del analito se pierde (sales insolubles). A partir de lo anterior se deduce que es el ácido idóneo para solventar la problemática detectada con los metales pesados analizados por las técnicas de absorción atómica horno de grafito y generador de hidruros (Skoog et al., 2015).

\section{REFERENCIAS}

Acosta, Y., Flores, S., Senior, A., \& Toyo, L. (2006). Evaluación de metales pesados en tres residuos orgánicos de diferente naturaleza. Laboratorio de Investigaciones y Servicios Ambientales (LISA), Centro de Ecología. Venezuela: Universidad del Zulia.

Agroanálisis Costa Rica. (2016). Agroanálisis. Obtenido de Agroanálisis: http://www.agroanalisiscr.com/

Aguilar, A., \& Navarro, J. (2007). Aspectos bioquímicos y genéticos de la tolerancia y acumulación de metales pesados en plantas. Revista Científica y Técnica de Ecología y Medio Ambiente, 10-24.

Alvarado, A., \& Romero, R. (2010). Acumulación de cobre en plantas silvestres de zonas agrícolas contaminadas con el metal. San José, Costa Rica: UCR.

Arderiu, H. (1997). Bioquímica clínica y patología molecular (Segunda edición ed., Vol. I). Barcelona, España: Reverte.

Ávila, E., Lira, J., \& Padilla, P. (1997). Metodología en línea para la determinación de trazas de los herbicidas 2,4-DY 2,4-DB en agua. Int. Contarn. Ambient, 63-71.

Ayala, J., Garro, O., \& Gimenez, M. (1999). Extracción Enzimática de Arsénico en Muestras de Orígen Vegetal. El Chaco, Argentina: UNNE.

Burba, J., Cavagnaro, M., \& Martí, L. (2010). Metales Pesados en Fertilizantes Fosfatados, Nitrogenados y Mixtos. Revista de la Facultad de Ciencias Agrarias, 43-48.

Casanova, A. (2007). Comparación de corrección teórica de efecto matriz, análisis de interferencias y corrección por compensación para equipos portátiles de fluoresencia de rayos x en minerales y suelos. Laboratorio de Química Ambiental, Departamento de Química Inorgánica y Analítica. Santiago: Universidad de Chile.

Finck, A. (2000). Fertilizantes y fertilización: fundamentos y métodos para la fertilización de los cultivos (Ilustrada ed.). Madrid, España: Reverte.

Gómez, D. (2011). Validación de la metodología para el análisis de metales pesados en muestras de aguas y aguas residuales por la técnica de absorción atómica. Universidad Tecnológica de Pereira, Departamento de Tecnología Química. Bogotá: CARDER.

Gómez, S., Pérez, J., \& Sierra, I. (2009). Analisis Instrumental (Vol. I). Madrid, España: NETBIBLO.

González, C., Prieto, J., Román, A., \& Solís, M. (2009). Contaminación y Fitotoxicidad en Plantas por Metales Pesados Provenientes de Suelo y Agua. Tropical and Subtropical Agroecosystems, 29-44. 
Guerrero, J. (2010). Estudio del efecto matriz en el análisis de plaguicidas y agroquímicos por la técnica de cromatografía de gases. Revista de la Facultad de Química Farmacéutica, 50-58.

Harris, D. (2006). Análisis químico cuantitativo (Reimpresa ed.). Madrid, España: Reverte.

Herrera, J., \& Román, S. (2010). Validación de un método de análisis para la determinación de metales pesados en partículas pm 10 colectadas en aire ambiente. Tecnología en Marcha, 33-46.

Laínez, J. (2005). Fertilización Química de Café y Cacao en el Litoral Ecuatoriano. (I. N. Agropecuarias, Ed.) Quito, Ecuador: INIAP.

Lázaro, J. (2008). Fitocorreción de suelos contaminados con metales pesados: evaluación de plantas tolerantes y optimización del proceso mediante prácticas agronómicas. (U.S. Compostela, Ed.) Coruña, España: USC.

Manzano, J. (2003). Calificación de operación de espectrofotómetros de absorción atómica. Querétaro, México: Centro Nacional de Metrología.

Morel, P. (2003). Tecnología de los fertilizantes (Segunda ed.). Santiago, Chile: Editorial Andrés Bello.

Navarro, M. (2014). Fertilizantes: química y acción (Vol. II). Murcia, España: Ediciones Paraninfo.

Ramos, J. (2009). Estudio de la contaminación por metales pesados y otros procesos de degradación química en los suelos en los invernaderos del poniente almeriense (Vol. XII). Almería, España: Universidad Almería.
Remache, A. M. (2013). Validación de método para análisis de metales pesados de diferentes matrices de suelos. Facultad de Ingeniería Química. Quito: Universidad Central del Ecuador.

Reyes, J., \& Walton, G. (2001). Análisis químico e instrumental moderno (Segunda ed.). Barcelona, España: Reverte.

Robledo, M. (2012). Validación de la Metodología para el Análisis de los Metales Cadmio y Plomo en Agua Tratada por Absorción Atómica con Horno de Grafito. Bogotá, Colombia: Universidad Tecnológica de Pereira.

Roma, M. (2002). Los Fertilizantes y Su Uso (Cuarta ed.). Madrid, España: FIAT PANIS.

Sandoval, S. (2010). Validación de métodos y determinación de la incertidumbre de la medición. (B. Duffau, F. Rojas, \& M. Soto, Edits.) Santiago, Chile: Instituto de Salud Pública.

Saucedo, L., Sierra, J., Zaldívar, M., \& Zúñiga, A. (2005). Validación de un método de determinación de $\mathrm{Na}, \mathrm{K}, \mathrm{Cd}, \mathrm{Pb}$ y $\mathrm{Hg}$ por Espectrometría de Absorción Atómica en policosanol y extracto purificado de cera de caña. CENIC , 9-14.

Skoog, D., Holler, J., \& West, H. (2015). Fundamentos de química analítica (Novena edición ed.). México: CENGAGE Learning.

Thompson, L., \& Trohe, F. (2007). Los suelos y su fertilidad (Reimpresa ed., Vol. I). Barcelona, España: Reverte.

Zúñiga, F. (2005). Introducción al estudio de la contaminación del suelo por metales pesados (Ilustrada ed., Vol. I). Yucatán, México: UADY. 